\title{
Carnitine in the Skeletal Muscle: Beyond Fatty Acid Metabolism
}

Yasuro Furuichi and Nobuharu L. Fujii*

Department of Health Promotion Sciences, Graduate School of Human Health Sciences, Tokyo Metropolitan University, 1-1 Minami-Osawa, Hachioji, Tokyo, Japan ${ }^{*}$ Corresponding author: Nobuharu L. Fuji, Department of Health Promotion Sciences, Tokyo Metropolitan University, Minami-Osawa, Hachioji, Tokyo, 193-0397, Japan, Tel: +81- 42-677-2966; Fax: +81- 42-677-2961; E-mail: fujiin@tmu.ac.jp

Rec date: June 21, 2016; Acc Date: July 07, 2016; Pub Date: July 11, 2016

Copyright: () 2016 Furuichi Y, et al. This is an open-access article distributed under the terms of the Creative Commons Attribution License, which permits unrestricted use, distribution, and reproduction in any medium, provided the original author and source are credited.

\section{Mini Review:}

Carnitine, or 3-hydroxy-4-trimethylammonium butyrate, was initially discovered in the muscle tissue in 1905 , and was subsequently identified as a fatty acid carrier in the mitochondrial membrane around 60 years later [1]. Since the skeletal muscle is the largest organ that utilizes fatty acids for energy production, carnitine has been a hot topic of research in the fields of internal medicine and sports science. Many researchers have proposed that carnitine supplementation improves skeletal muscle metabolism in healthy individuals by promoting increased fatty acid utilization. However, there is no convincing evidence demonstrating that carnitine supplementation has an enhancing effect on human metabolism and exercise performance [2]. Nevertheless, recent studies have shown that carnitine plays other novel roles in the skeletal muscle.

Studies conducted in the last decade have highlighted another role of carnitine in buffering the excess mitochondrial acetyl-CoA, an intermediate metabolite of beta-oxidation and glycolytic metabolism. Although acetyl-CoA is an essential metabolite, excess accumulation of acetyl-CoA inhibits pyruvate dehydrogenase (PDH), a rate-limiting enzyme for pyruvate entry into the tricarboxylic acid cycle, resulting in negative regulation of glucose uptake. In this situation, carnitine binds to acetyl-CoA and is converted to acetylcarnitine, resulting in a decrease in acetyl-CoA levels. This reaction is induced by carnitine acetyltransferase (CrAT), which is present in the mitochondrial matrix. Muscle-specific CrAT knockout mice showed glucose intolerance and insulin resistance [3], providing a direct demonstration of the significance of CrAT in glucose metabolism. Importantly, carnitine supplementation for 6 months in glucose-intolerant human subjects decreased plasma glucose and insulin levels, and improved insulin resistance, demonstrating that carnitine has therapeutic potential in diabetic patients [3]. Taken together, the evidence collected thus far suggests that CrAT plays a role in the maintenance of energy production by relieving acetyl-CoA accumulation in the skeletal muscle.

Glucose and fatty acids utilization is acutely enhanced by exercise (muscle contraction), which leads to the accumulation of acetyl-CoA in the skeletal muscle cells. In this situation, the acetyl-CoA concentration is regulated by carnitine, which is converted to acetylcarnitine. We recently visualized the distribution of endogenous acetylcarnitine in the rat skeletal muscle using matrix-assisted laser desorption/ionization imaging mass spectrometry, which is a novel two-dimensional mass spectrometry technique [4]. The acetylcarnitine signal intensity was increased by acute muscle contraction, and its localization corresponded to regions of glycogen depletion, suggesting that carnitine acetylation is dependent on contractile activity.

Interestingly, recent studies using cell culture experiments demonstrated that acetylcarnitine is exported from skeletal muscle cells [5]. However, acetylcarnitine and carnitine play different roles. For example, acetylcarnitine is a potentially beneficial molecule in the treatment of various neurological diseases such as Alzheimer's disease [6]. Acetylcarnitine exported from skeletal muscle cells potentially affects various organs in the body, implying the inter-organ shuttling of carnitine systems (Figure 1).

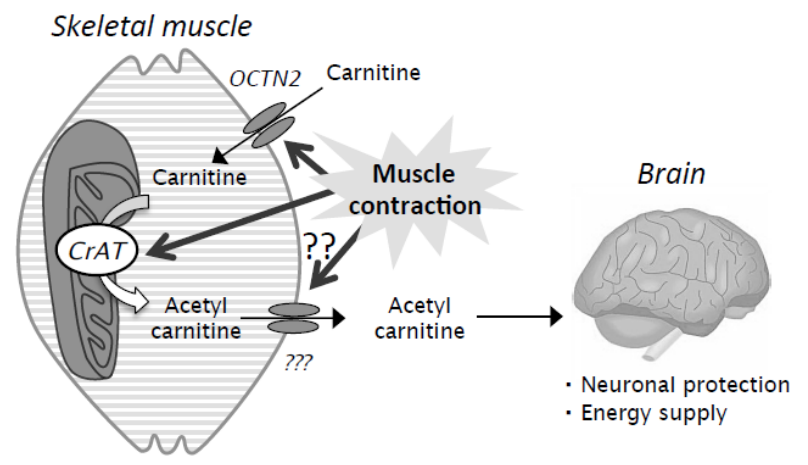

Figure 1: Carnitine dynamics during muscle contraction. When energy production exceeds the capacity of mitochondrial utilization, carnitine is converted to acetylcarnitine by the mitochondrial enzyme CrAT. The acetylcarnitine produced is then exported from the skeletal muscle cell into the bloodstream. Muscle contraction increases both carnitine uptake and its acetylation. It is hypothesized that the acetylcarnitine produced during muscle contraction is exported to plasma and affects various organs such as the brain.

We hypothesized that muscle contraction-induced acetylcarnitine production is not only beneficial for buffering the excess acetyl-CoA but also has other health effects in an endocrine manner. In fact, previous work has shown that the plasma acetylcarnitine concentration was increased during human exercise [7], suggesting that the acetylcarnitine produced during muscle contraction may be released from muscle cells to serve different functions. We have shown that carnitine uptake from the blood to the skeletal muscle is acutely regulated by muscle contraction in a rat in situ model via the carnitine transporter OCTN2, suggesting that carnitine transport across the skeletal muscle plasma membrane is dynamically controlled during exercise $[8,9]$. The mechanisms regulating the export of acetylcarnitine during muscle contraction and its physiological significance remain to be elucidated in future. 


\section{References}

1. Bremer J (1962) Carnitine as a fatty acid carrier in intermediary metabolism. Nature 196: 993-994.

2. Brass EP (2004) Carnitine and sports medicine: use or abuse? Ann N Y Acad Sci 1033: 67-78.

3. Muoio DM, Noland RC, Kovalik JP (2012) Muscle-specific deletion of carnitine acetyltransferase compromises glucose tolerance and metabolic flexibility. Cell Metab 15: 764-777.

4. Furuichi Y, Goto-Inoue N, Manabe Y, Setou M, Masuda K, et al. (2014) Imaging mass spectrometry reveals fiber-specific distribution of acetylcarnitine and contraction-induced carnitine dynamics in rat skeletal muscles. Biochim Biophys Acta 1837: 1699-1706.

5. Noland RC, Koves TR, Seiler SE, Lum H, Lust RM, et al. (2009) Carnitine insufficiency caused by aging and overnutrition compromises mitochondrial performance and metabolic control. J Biol Chem 284: 22840-22852.

6. Pettegrew JW, Levine J, McClure RJ (2000) Acetyl-L-carnitine physicalchemical, metabolic, and therapeutic properties: relevance for its mode of action in Alzheimer's disease and geriatric depression. Mol Psychiatry 5: 616-632.

7. Vukovich MD, Costill DL, Fink WJ (1994) Carnitine supplementation: effect on muscle carnitine and glycogen content during exercise. Med Sci Sports Exerc 26: 1122-1129.

8. Furuichi Y, Sugiura T, Kato Y, Shimada Y, Masuda K (2010) OCTN2 is associated with carnitine transport capacity of rat skeletal muscles. Acta Physiol (Oxf) 200: 57-64.

9. Furuichi Y, Sugiura T, Kato Y, Takakura H, Hanai Y, et al. (2012) Muscle contraction increases carnitine uptake via translocation of OCTN2. Biochem Biophys Res Commun 418: 774-779. 